\title{
Transcription and transcript processing in the sdhCDAB-suCABCD operon of Escherichia coli
}

\author{
Louise Cunningham and John R. Guest
}

The Krebs Institute for Biomolecular Research, Department of Molecular Biology and

Biotechnology, University of Sheffield, Western Bank, Sheffield S10 2TN UK
Author for correspondence: John R. Guest. Tel: +44114222 4406/3. Fax: +44114272 8697. e-mail : j.r.guest@sheffield.ac.uk

The genes encoding succinate dehydrogenase (sdhCDAB), the specific components of the 2-oxoglutarate dehydrogenase complex (ODH, E10 and E20; suCAB) and succinyl-COA synthetase (suCCD) form a cluster containing two promoters at $16.3 \mathrm{~min}$ in the chromosome of Escherichia coli: $\boldsymbol{P}_{\text {sdh }}$ SdhCDAB-P $\boldsymbol{P}_{\text {suc }}$ sUCAB-suCCD. The gene encoding the lipoamide dehydrogenase component of both the 2-oxoglutarate and pyruvate dehydrogenase complexes (E3; IPdA) is the distal gene of another cluster containing two promoters located at $2.7 \mathrm{~min}$ : $P_{\text {pdh }}$ PdhR-aceEF- $\boldsymbol{P}_{\text {lpd }} I$ IPdA. The responses of the suc and Ipd promoters to different environmental conditions and to regulator defects were investigated with appropriate lacz fusions, in order to understand how expression of the suCAB genes is co-regulated with other genes in the sdhCDAB-suCABCD cluster and with IpdA expression. Expression from the suc promoter was repressed by IHF and partially activated by $\sigma^{38}$ but it was not regulated by ArCA, FNR, CRP, FruR or Fis, and not repressed by glucose or anaerobiosis, indicating that the well-established catabolite and anaerobic repression of ODH synthesis is imposed elsewhere. In contrast, the Ipd promoter was repressed by both glucose (via a CRP-independent mechanism) and anaerobiosis (mediated by ArCA), and activated by Fis, but it was not regulated by FNR, FruR, IHF or $\sigma^{38}$. These observations support the view that transcription of the sUCABCD genes is primarily initiated and regulated at the upstream $s d h$ promoter, and that the Ipd promoter is independently co-regulated with $\boldsymbol{P}_{\text {seh }}$ (primarily by ArcAmediated repression) rather than with $\boldsymbol{P}_{\text {suc }}$. Direct evidence for co-transcription of the entire sdhCDAB-suCABCD region from $P_{\text {solh }}$ was obtained by detecting a $10 \mathrm{~kb}$ transcript in rnc and rne mutants, but not in the parental strains. Three RNaselll-specific processing sites, which contribute to the extreme instability of the readthrough transcript, were identified in the sdhCDAB-suCABCD intergenic region. Other sites of endonuclease processing were located by interpreting the patterns of transcript subfragments observed in Northern blotting.

Keywords: 2-oxoglutarate dehydrogenase, succinate dehydrogenase, lipoamide dehydrogenase, mRNA processing, RNaseIII

\section{INTRODUCTION}

The pyruvate dehydrogenase and 2-oxoglutarate dehydrogenase (PDH and $\mathrm{ODH}$ ) complexes are analogous multienzyme complexes that catalyse the oxidative decarboxylation of pyruvate to acetyl-CoA or of 2-

Abbreviations: CRP, CAMP receptor protein; FNR, fumarate and nitrate reduction regulator; IHF, integration host factor; $\mathrm{ODH}$, 2-oxoglutarate dehydrogenase; PDH, pyruvate dehydrogenase; SCS, succinyl-COA synthetase; SDH, succinate dehydrogenase. oxoglutarate to succinyl-CoA (Guest et al., 1989; Perham, 1991). They perform important metabolic roles in funnelling glycolytic carbon into the citric acid cycle and its subsequent oxidation in the cycle. The synthesis of the complexes under different environmental conditions has been extensively studied at the enzyme and protein levels (Amarasingham \& Davis, 1965; Buck et al., 1986; Quail et al., 1994; Smith \& Niedhardt, 1983). Thus, the PDH complex is induced by pyruvate and partially repressed by growth on glucose or acetate and by anaerobiosis. In contrast, the ODH complex is most 

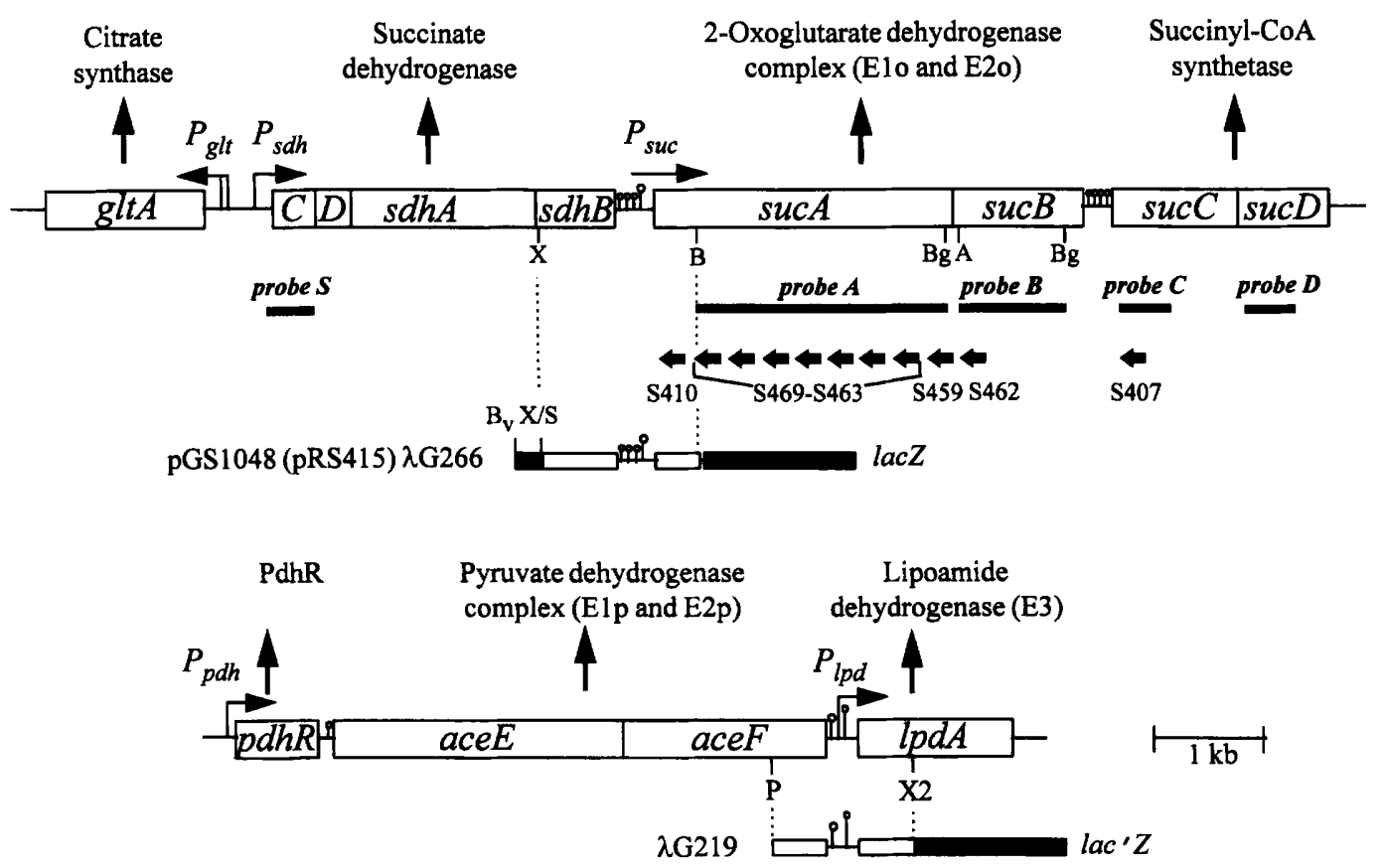

Fig. 1. Organization of a gene cluster encoding four citric acid cycle enzymes (gltA-sdhCDAB-sucABCD; $16.3 \mathrm{~min}$ ) and the pyruvate dehydrogenase operon (pdhR-aceEF-lpdA; $2.7 \mathrm{~min})$. The positions and polarities of relevant promoters are marked by arrows, and the IRU (ERIC), REP and other significant potential stem-loop sequences are also indicated ( $($ ). The DNA fragments used to make lacZ fusions, the positions of specific gene probes $(S, s d h ; A, \operatorname{sucA} ; B, \operatorname{sucB} ; C$, sucC; and $D$, suCD), and the oligonucleotides used in primer extension analysis (arrows), are also indicated. Relevant restriction sites are shown: A, Avall; B, BamHI; Bg, Bg/ll; P, Pstl; S, Sall; X, Xhol; and X2, Xholl (B, refers to a flanking vector site).

highly induced during aerobic growth on acetate and citric acid cycle intermediates, and like succinate dehydrogenase and succinyl-CoA synthetase (SCS) it is severely repressed by excess glucose, anaerobiosis and cAMP deficiency. Indeed, the repression of these three co-ordinately regulated enzymes is a major feature of the conversion of the citric acid cycle into its branched or non-cyclic anaerobic form (Guest \& Russell, 1992; Cronan \& LaPorte, 1996).

The PDH and ODH complexes are encoded by two distinct gene clusters in the Escherichia coli chromosome (Fig. 1). The $p d h$ operon ( $p d b R-a c e E F-l p d A$ ) at $2.7 \mathrm{~min}$ encodes a pyruvate-responsive repressor (PdhR), the specific pyruvate dehydrogenase (E1p) and lipoate acetyltransferase (E2p) components of the PDH complex, and lipoamide dehydrogenase (E3), which is a common component of both the $\mathrm{PDH}$ and $\mathrm{ODH}$ complexes (Quail et al., 1994). The genes are expressed from two major promoters: $P_{p d h}$, which generates a $7.4 \mathrm{~kb} p d b R-l p d A$ readthrough transcript; and $P_{l p d}$, which generates an independent $1.7 \mathrm{~kb} l p d A$ transcript (Quail et al., 1994; Spencer \& Guest, 1985). Quantitative S1 mapping studies indicated that transcription from $P_{l p d}$ is co-regulated with synthesis of the ODH complex, so as to maintain a supply of E3 subunits when the synthesis of all components of the PDH complex (via $P_{p d h}$ ) is repressed, but synthesis of the ODH complex is induced (Spencer \& Guest, 1985). The specific dehydrogenase and lipoate succinyltransferase com- ponents of the ODH complex are encoded by the sucA (E1o) and sucB (E2o) genes in the gltA-sdbCDABsucAB-sucCD gene cluster at $16.3 \mathrm{~min}$ (Fig. 1), which additionally encodes citrate synthase (CS, glt $A$ ), succinate dehydrogenase (SDH, sdhCDAB), and succinylCoA synthetase (SCS, sucCD). All of the genes, except glt $A$, have the same polarity, and S1 mapping studies established that the $g l t A-s d h A$ intergenic region contains two gltA promoters and a divergent but nonoverlapping $s d h$ promoter, $P_{s d h}$ (Wilde \& Guest, 1986). Similar studies by Spencer \& Guest (1985) showed that there is a single suc promoter $\left(P_{\text {suc }}\right)$ upstream of the $s u c A$ gene and that transcription continues between the $s u c A B$ and sucCD genes: no independent transcription of the sucCD genes was detected (Fig. 1). Transcript termination (or processing) sites were identified immediately downstream of the $s d h C D A B$ and $s u c A B$ genes, but significantly, no transcript linking the $s d b B$ and sucA genes was detected (Spencer \& Guest, 1985). This suggested that the ODH complex and SCS are coexpressed independently of SDH. The lpdA gene is preceded by an IRU (ERIC) sequence and the sucAB genes are flanked by three and four REP sequences, but it is not known whether these have any effect on transcription.

Early studies with galK transcriptional fusions indicated that $P_{s d h}$ and $P_{l p d}$ are more active than $P_{s u c}$, and that $P_{s d h}$ is strongly repressed by anaerobiosis and repressed fivefold both by glucose and by crp cya double deletion, 
whereas $P_{s u c}$ and particularly $P_{l p d}$ are much less affected by such conditions; $P_{s u c}$ and $P_{l p d}$ also differ in lacking potential CRP-binding sites (Spencer et al., 1986; Spencer \& Guest, 1987; Guest et al., 1989). Indeed, to account for the discrepancies between the patterns of $P_{s u c}$ activity and the corresponding ODH and SCS enzyme activities, it was suggested that the sucABCD genes may normally be transcribed and regulated from the $s d h$ promoter, despite the lack of evidence for $s d h-s u c$ readthrough transcription. More recently, studies with lacZ fusions have shown that $s d h$ gene expression is anaerobically repressed some 70 -fold by the combined effects of ArcA and FNR, and repressed up to threefold by glucose in a CRP- (and FruR-) independent manner (Iuchi et al., 1994; Park et al., 1995). In contrast, the $p d h$ promoter is not strongly controlled by ArcA, FNR or CRP (Quail et al., 1994).

The present work was initiated to investigate the effects of global regulators on the suc and $l p d$ promoters, in order to understand how expression of the $s u c$ and $l p d$ genes might be co-regulated. The results support the view that the suc genes are transcribed and regulated primarily from the $s d h$ promoter and that co-expression of the $s d h, s u c$ and $l p d$ genes is mediated by ArcA. Direct evidence that the $s d h$ and $s u c$ genes belong to a single operon transcribed primarily from the $s d h$ promoter was obtained by detecting an $s d h C D A B-s u c A B C D$ readthrough transcript in $r n c$ and $r n e$ mutants.

\section{METHODS}

Bacterial strains, plasmids and bacteriophages. The strains of Escherichia coli $\mathrm{K}-12$ are listed in Table 1: DH5 $\alpha$ was the routine transformation host; MC4100 was the primary host for expression studies with lac $Z$ fusions; and several strains, including W3110, RS6521 ( rnc) and N3431 (rne ${ }^{\text {ts }}$ ), were used as RNA sources. A sucA-lacZ transcriptional fusion was constructed by cloning the $1.65 \mathrm{~kb} B a m H I$ fragment containing the $s d h B-s u c A$ intergenic region and a small segment of vector DNA from pGS128 into the lacZ fusion vector (pRS415) to produce pGS1048 and ultimately $\lambda$ G266 (Fig. 1). The orientation of the subcloned BamHI fragment was established by defining the location of an asymmetric PstI site. An analogous sucA-lac $Z$ translational fusion was constructed by cloning the same fragment 'in phase' into pMD1406, to generate pGS982 and ultimately $\lambda \mathrm{G} 264$ (Table 1). Transfer of fusions to $\lambda R Z 5$ and the construction of monolysogens was described previously (Quail et al., 1994). The $\lambda l p d-l a c Z$ translational fusion phage, $\lambda$ G219 (Fig. 1), was constructed previously (Quail et al., 1994).

Monolysogens of MC4100 containing each of the fusion phages were constructed according to Cunningham et al. (1997) and further derivatives containing $\operatorname{arcA}, \mathrm{fnr}, \mathrm{fruR}$, $\operatorname{bim} A, \operatorname{rpos}$ and fis mutations were constructed by P1 transduction using the corresponding donor strains (Table 1). In the case of crp, the single copies of the fusion prophages were first established in JRG1999(pGS279), $\Delta$ lac $\Delta c r p\left(c r p^{+}\right)$, from which the plasmid was subsequently cured. Appropriate multicopy plasmids were used to complement the arcA (pRB38), fnr (pCH21) and crp (pGS279) mutations in the corresponding monolysogenic derivatives.

Microbiology and enzymology. Bacteria were cultured in $\mathrm{L}$ broth with glucose $(0.4 \%$ or $1 \%)$ where stated, and ap- propriate antibiotics $\left(\mu \mathrm{g} \mathrm{ml}^{-1}\right)$ : ampicillin, 100 ; kanamycin, 50; chloramphenicol, 25 ; tetracycline, 15 . The minimal salts medium, M9 (Sambrook et al., 1989), was supplemented with glucose $(11 \mathrm{mM})$ or with sodium acetate, sodium pyruvate or sodium 2-oxoglutarate (each at $40 \mathrm{mM}$ ) as carbon sources, and thiamin hydrochloride $\left(5 \mu \mathrm{g} \mathrm{ml}^{-1}\right)$. For expression studies, monolysogens containing $\lambda$ lac $Z$ fusion prophages were grown either aerobically in shaken flasks or under $\mathrm{H}_{2}$ and $\mathrm{CO}_{2}(95: 5)$ in anaerobic jars. The $\beta$-galactosidase specific activities [nmol ONPG hydrolysed $\mathrm{min}^{-1}$ (mg protein $)^{-1}$ ] were determined for permeabilized cells (Miller, 1972) using a Labsystems iEMS plate-reader and flat-bottomed microtitre plates to monitor changes in $A_{420}$ and to measure protein concentrations (Philips-Jones et al., 1993; Bradford, 1976). The quoted specific activities refer to exponential-phase cultures $\left\langle\mathrm{OD}_{600}\right.$ $=1.0$ for $\mathrm{L}$ broth, 0.3 for acetate minimal medium, and 0.8 for all other minimal media); they were averaged from triplicate samples from three independent cultures, the overall variation being $<20 \%$.

DNA manipulation, RNA extraction, Northern hybridization and primer extension analysis. DNA was prepared and manipulated by standard procedures (Sambrook et al., 1989). RNA was extracted by the hot acid phenol procedure (Aiba et al., 1981) from exponential-phase cultures $\left(\mathrm{OD}_{600}=0.6\right)$ that had been rapidly cooled to $4{ }^{\circ} \mathrm{C}$ in liquid $\mathrm{N}_{2}$. The cultures were grown aerobically in $\mathrm{L}$ broth at $37^{\circ} \mathrm{C}$ except for the $r n e^{\text {ts }}$ mutant and its parent, which were grown at $30^{\circ} \mathrm{C}$ to $\mathrm{OD}_{600}$ 0.6 and then given a temperature shock $\left(4 \mathrm{~min}\right.$ at $\left.43^{\circ} \mathrm{C}\right)$ to destroy RNaseE activity. Bacteria equivalent to $50 \mathrm{ml} \mathrm{ex-}$ ponential culture $\left(\mathrm{OD}_{600}=0.6\right)$ were resuspended in $3 \mathrm{ml}$ $20 \mathrm{mM}$ sodium acetate ( $\mathrm{pH} 5.5$ ) containing $0.5 \%$ SDS and $1 \mathrm{mM}$ EDTA, and then extracted at $60^{\circ} \mathrm{C}$ with phenol (equilibrated with $20 \mathrm{mM}$ sodium acetate, $\mathrm{pH} 5 \cdot 5$ ). The aqueous phase was reextracted with phenol, and the RNA was recovered by ethanol precipitation and dissolved in diethylpyrocarbonate-treated water after two further ethanol precipitations.

Northern hybridization was performed after transferring denatured and electrophoretically fractionated RNA samples ( $20 \mu \mathrm{g}$ in $1 \%$ agarose formaldehyde gels) to nylon membranes, using $\left[\alpha^{32} \mathrm{P}\right] \mathrm{dCTP}$-labelled probes according to the 'Ready to Go' labelling-kit instructions (Pharmacia). The sucA probe was a $2 \cdot 2 \mathrm{~kb} B$ am $\mathrm{HI}-B g l \mathrm{II}$ fragment of pGS128 (probe $\boldsymbol{A}$; Fig. 1) and the $s u c B$ probe was a $0.9 \mathrm{~kb}$ AvalI-BgllI fragment of pGS128 (probe B; Fig. 1). Specific probes for $s d h$, sucC and $s u c D$ regions (probes $S, C$ and $D$, respectively; Fig. 1), were generated by PCR amplification using the following DNA templates and oligonucleotide primers (co-ordinates from GenBank accession number J01619): probe $S, \lambda G 118$ with S451 (3061-3078) and S452 (3378-3349); probe C, pGS128 with S453 (10921-10938) and S454 (11449-11432); and probe $D, \lambda$ G118 with S455 (12117-12125) and S456 (12693-12676). RNA stability was investigated by growing cultures to $\mathrm{OD}_{600}$ $0 \cdot 6$, adding rifampicin $\left(200 \mu \mathrm{g} \mathrm{ml} \mathrm{m}^{-1}\right)$, and thereafter withdrawing samples $(10 \mathrm{ml})$ at 1 min intervals. These were chilled immediately to $4{ }^{\circ} \mathrm{C}$ with liquid nitrogen and the RNA was isolated and used in Northern hybridization (as above) with $s d h$, sucA and $s u c B$ probes. The autoradiographs were analysed using a Bio-Rad 690 densitometer.

Primer extension analysis was performed by the method of Gerischer \& Dürre (1992), modified to allow continuous incorporation of $\left[\alpha^{-32} \mathrm{P}\right] \mathrm{dCTP}$, as described by Cunningham $e t$ al. (1997). Samples of total RNA $(100 \mu \mathrm{g})$ from cultures of W3110, harvested at $\mathrm{OD}_{600} 0 \cdot 5-0 \cdot 6$, were used with $10 \mathrm{pmol}$ primer. After processing, the samples were fractionated by electrophoresis in $6 \%$ acrylamide $/ 7 \mathrm{M}$ urea gels alongside a 
Table 1. E. coli strains, plasmids and phages

\begin{tabular}{|c|c|c|}
\hline $\begin{array}{l}\text { Strain, plasmid } \\
\text { or phage }\end{array}$ & Relevant characteristics & Source or reference* \\
\hline W3110 & Prototroph & Laboratory collection \\
\hline DH5 $\alpha$ & $\Delta($ argF-lac $) U 169(\phi 80 \Delta l a c Z M 15)$ recA & Sambrook et al. (1989) \\
\hline P90C & $\Delta($ lac-pro $)$ ara thi gly $A:: \operatorname{Tn} 5$ & Miller \& Albertini (1983) \\
\hline RS6521 & P90C rnc: : Tn10 & Miller \& Albertini (1983) \\
\hline N3433 & pyrC46 & Goldblum \& Apirion (1981) \\
\hline N3431 & N3433 $r n e^{\mathrm{ts}}$ & Goldblum \& Apirion (1981) \\
\hline MC4100 & $\Delta(\arg F-l a c) U 169 r p s L$ & Silhavy et al. (1984) \\
\hline ECL585 & $\operatorname{arc} z j j:: \operatorname{Tn} 10$ & Iuchi \& Lin (1988) \\
\hline LCB261 & thi thr leu lacY ara rpsL fnr-22 zcj:: Tn10 & Bonnefoy et al. (1986) \\
\hline JRG3227 & $\Delta f r u R:: k a n^{\mathrm{R}}$ & Cunningham et al. (1997) \\
\hline RJ1802 & fis-767 $\left(n e o^{\mathrm{R}}\right)$ & Ball et al. (1992) \\
\hline RH90 & MC4100 rpoS359:: Tn10 & R. Hennge-Aronis ${ }^{1}$ \\
\hline SJP3 & $\operatorname{bim} A:: \operatorname{Tn} 10$ & Schröder et al. (1993) \\
\hline JRG1999 & MC1000 $\Delta l a c X 74 \Delta($ araA-leu $) \Delta c r p T 8$ & S. T. Cole ${ }^{2}$ \\
\hline pGS128 & $\operatorname{sucABC} C^{+}, \mathrm{Ap}^{\mathrm{R}}$ & Buck et al. (1986) \\
\hline pRS415 & lacZ transcriptional fusion vector & Simons et al. (1987) \\
\hline pGS1048 & $\mathrm{pRS} 415, s d b^{\prime} B-s u c A^{\prime}, \mathrm{Ap}^{\mathrm{R}}$ & This work \\
\hline pMD1406 & lacZ translational fusion vector & Buck et al. (1987) \\
\hline pGS982 & $\mathrm{pMD} 1406, s d h^{\prime} B-s u c A^{\prime}, \mathrm{Ap}^{\mathrm{R}}$ & This work \\
\hline pRB38 & $\operatorname{arcA} A^{+}, \mathrm{Cm}^{\mathrm{R}}$ & R. S. Buxton ${ }^{4}$ \\
\hline $\mathrm{pCH} 21$ & $f n r^{+}, \mathrm{Ap}^{\mathrm{R}}, \mathrm{Cm}^{\mathrm{R}}$ & C. F. Higgins ${ }^{3}$ \\
\hline pGS279 & $c r p^{+}, \mathrm{Ap}^{\mathbf{R}}, \mathrm{Cm}^{\mathrm{R}}$ & Spiro et al. (1989) \\
\hline$\lambda \mathrm{G} 118$ & $\lambda g l t A-s d h C D A B-s u c A B C D-f a r R$ & Spencer \& Guest (1982) \\
\hline$\lambda \mathrm{G} 219$ & $\lambda l p d A-l a c^{\prime} Z$ translational fusion; $A^{R}$ & Quail et al. (1994) \\
\hline$\lambda \mathrm{G} 264$ & $\lambda s u c A-l a c^{\prime} Z$ translational fusion; $A^{R}$ & This work \\
\hline$\lambda G 266$ & $\lambda s u c A-l a c Z$ transcriptional fusion; $A^{\mathbf{R}}$ & This work \\
\hline
\end{tabular}

* 1, University of Konstanz, Germany; 2, Pasteur Institute, Paris, France; 3, Imperial Cancer Research Fund, John Radcliffe Hospital, Oxford, UK; 4, National Institute for Medical Research, Mill Hill, London, UK.

sequence ladder derived from the corresponding DNA and primer. The oligonucleotide primers were (co-ordinates from GenBank accession number J01619): for sucA, S410 (66386609), S463 (9205-9183), S464 (8933-8910), S465 (8664-8641), S466 (8421-8401), S467 (8160-8141), S468 (7914-7893) and S469 (7646-7621); for sucB, S459 (9493-9467) and S462 (9752-9728); and for sucC, S407 (10944-10915).

Materials. Restriction enzymes were from Northumbria Biologicals. AMV reverse transcriptase was from Life Sciences and the RNA calibration ladder was from Life Technologies. Radiolabelled $\left[\alpha_{-}^{-32} \mathrm{P}\right] \mathrm{dCTP}\left(110 \mathrm{TBq} \mathrm{mmol}^{-1}\right)$ and nylon membranes were from Amersham.

\section{RESULTS}

\section{Studies with transcriptional and translational fusions}

The existence of an independent sucABCD promoter $\left(P_{\text {suc }}\right)$ was originally inferred because the suc genes are expressed from subcloned fragments of the $s d h C D A B-$ sucABCD region, and this was confirmed in early studies with a sucA-galK transcriptional fusion. However, the activity of the suc promoter appeared not to be correlated with the synthesis of the ODH complex and SCS, particularly with respect to cAMP-dependent catabolite repression and anaerobic repression. The control of $P_{s u c}$ was accordingly reinvestigated with a series of monolysogens containing $\lambda s u c A-l a c Z$ translational and transcriptional fusions ( $\lambda \mathrm{G} 264$ and $\lambda 266$, respectively; see Methods and Table 1 ). The activity of $P_{s u c}$ was also compared with that of $P_{l p d}$ in an analogous series of $\lambda l p d A-l a c Z$ ( $\lambda G 219)$ monolysogens, in order to study the co-regulation of $s u c A B$ (E1o and E2o) and lpdA (E3) expression. The relative activities of the sucA-lacZ and $l p d A-l a c Z$ fusions in cultures sampled when the enzyme activities are at their highest are summarized in Tables 2 and 3 . The activities of the sucA-lacZ translational fusion were uniformly about 10-fold lower than those of the corresponding transcriptional fusion, and were not included in the Tables.

Regulation of sucA-lacz expression. The activity of the suc promoter was relatively unaffected by the growth substrate, the presence of glucose, or the absence of oxygen (Tables 2 and 3). The activity varied within a threefold range compared to an overall 70 -fold range for the corresponding enzymes (Buck et al., 1986). It is not known whether the 1.5-1.7-fold induction observed in 2oxoglutarate minimal medium compared to other mini- 
Table 2. Effects of growth substrate on sucA-lacZ and Ipd-lacZ expression

The $\beta$-galactosidase specific activities [nmol ONPG hydrolysed

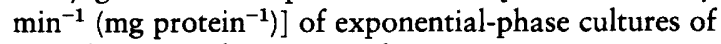
monolysogenic derivatives of MC4100 containing $\lambda$ sucA-lacZ transcriptional or $\lambda l p d A-l a c Z$ translational fusion phages are shown (see Methods). The overall variation between triplicate samples from three independent cultures was $<20 \%$.

\begin{tabular}{|lcc|}
\hline $\begin{array}{l}\text { Growth } \\
\text { substrate }\end{array}$ & \multicolumn{2}{c|}{$\boldsymbol{\beta}$-Galactosidase specific activity } \\
\cline { 2 - 3 } & $\begin{array}{c}\text { sucA-lacZ } \\
(2 \mathrm{G} 266)\end{array}$ & $\begin{array}{c}\text { lpdA-lacZ } \\
(2 \mathrm{G} 219)\end{array}$ \\
\hline Glucose & 581 & 923 \\
Pyruvate & 581 & 2282 \\
Acetate & 513 & 2051 \\
2-Oxoglutarate & 863 & 2111 \\
L broth & 385 & 1183 \\
\hline
\end{tabular}

mal media (also seen with the enzymes), reflects the existence of a specific 2-oxoglutarate-responsive regulatory system. The effects of anaerobiosis and $\operatorname{arcA}$ or f $n r$ mutations on $P_{s u c}$ activity were also relatively small (Table 3) when compared to the eightfold repression of ODH activity that accompanies anaerobiosis, and the 1.7-fold derepression associated with $\operatorname{arcA}$ mutation
(Iuchi \& Lin, 1988). The main effect of $f n r$ was the two- to threefold anaerobic repression of $P_{s u c}$ activity imposed by multicopy $\mathrm{fnr}^{+}$(Table 3 ), which presumably has little relevance under physiological conditions. It was also observed that $c r p$ mutation increased $P_{s u c}$ activity in a manner that could be reversed by a multicopy $\mathrm{crp}^{+}$plasmid (Table 3). Thus, in complete contrast to the observed catabolite sensitivity of ODH synthesis, suc expression appears to be repressed rather than activated by CRP. The significance of these observations is obscure, especially as the repression was not modulated by glucose. The consequences of inactivating other potential regulators showed that IHF (the $\operatorname{bim} A$ gene product) performs a major role in repressing $P_{s u c}$ activity and that a significant amount of transcription from $P_{s u c}$ may be initiated by $\sigma^{38}$ (the $r p o S$ gene product). The effects of both of these mutations were sustained throughout the entire growth cycle. No regulation by FruR or Fis was detected (Table 3). The results agree with those of Park et al. (1997), who found that ArcA and FNR had no effect on the sucA promoter whereas its activity was markedly increased in a $\operatorname{him} A$ (IHF) mutant.

Regulation of IpdA-lacZ expression. The effects of different regulators and growth conditions on expression of the independent lpd promoter were investigated in an attempt to understand the mechanism of co-regulation with $s u c A B$ expression. No direct correlation between $P_{l p d}$ activity and lipoamide dehydrogenase (E3) enzyme

Table 3. Effects of mutations in global regulatory genes on sucA-lacZ and Ipd-lacZ expression

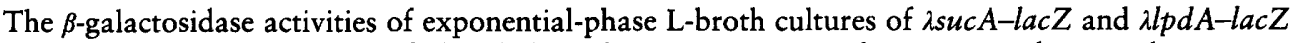
monolysogens containing specified global regulatory mutations \pm the corresponding regulatory plasmid (see Methods) are shown. The specific activities are expressed as percentages relative to the values for aerobic L-broth cultures of the parental strains $\left({ }^{*}\right), 385$ or $1183 \mathrm{nmol}$ ONPG hydrolysed $\mathrm{min}^{-1}$ (mg protein) ${ }^{-1}$, respectively, as shown in Table 2 . In each case the overall variation between triplicate samples from three independent cultures was $<20 \%$. The concentration of added glucose was $0.4 \%(\mathrm{w} / \mathrm{v})$ except in studies with the $c r p$ and $f r u R$ mutants $(\dagger)$, where it was $1.0 \%(\mathrm{w} / \mathrm{v})$. Increasing the glucose concentration had negligible effects on the parental strains. - , Not done.

\begin{tabular}{|c|c|c|c|c|c|c|}
\hline \multirow{3}{*}{$\begin{array}{l}\text { Strain or } \\
\text { genotype }\end{array}$} & \multicolumn{6}{|c|}{$\beta$-Galactosidase specific activity ( $\%$ of parental control) } \\
\hline & \multicolumn{3}{|c|}{$\operatorname{suc} A-\operatorname{lac} Z(\lambda \mathrm{G} 266)$} & \multicolumn{3}{|c|}{ lpdA-lacZ (גG219) } \\
\hline & $\begin{array}{c}\text { L } \\
\text { (aerobic) }\end{array}$ & $\begin{array}{c}\mathbf{L}+\text { glucose } \\
\quad \text { (aerobic) }\end{array}$ & $\begin{array}{l}\mathbf{L}+\text { glucose } \\
\text { (anaerobic) }\end{array}$ & $\begin{array}{c}\mathrm{L} \\
\text { (aerobic) }\end{array}$ & $\begin{array}{c}\mathrm{L}+\text { glucose } \\
\text { (aerobic) }\end{array}$ & $\begin{array}{l}\mathbf{L}+\text { glucose } \\
\text { (anaerobic) }\end{array}$ \\
\hline MC4100* & $100^{*}$ & 75 & 78 & $100^{*}$ & 63 & 33 \\
\hline $\operatorname{arcA}$ & 71 & 50 & 97 & 176 & 126 & 152 \\
\hline $\operatorname{arcA}+\mathrm{pRB} 38$ & 70 & 62 & 70 & 42 & 43 & 18 \\
\hline fnr & 88 & 80 & 93 & 105 & 74 & 31 \\
\hline$f n r+\mathrm{pCH} 21$ & 79 & 67 & 34 & 79 & 52 & 6 \\
\hline crp & 201 & $176+$ & - & 101 & $107 \dagger$ & - \\
\hline$c r p+p G S 279$ & 112 & $135 t$ & - & 77 & $58 t$ & - \\
\hline fruR & 105 & $75 \dagger$ & - & 108 & $45 \dagger$ & - \\
\hline fis & 120 & - & - & 47 & - & - \\
\hline rpos & 53 & - & - & 89 & - & - \\
\hline $\operatorname{bim} A$ & 299 & - & - & 90 & - & - \\
\hline
\end{tabular}


activity was expected because enzyme synthesis is controlled by transcription from $P_{p d h}$ as well as from $P_{l p d}$ (Fig. 1). The parental activity of $P_{l p d}$ varied over a sixfold range depending on the substrate and growth conditions, being lowest during anaerobic growth with glucose and highest with pyruvate under aerobic conditions (Tables 2 and 3). This pattern of induction during growth on non-fermentable substrates in minimal media, and repression by glucose and anaerobiosis, is what would be expected for a citric acid cycle promoter, rather than the pyruvate-induced and acetaterepressed profile associated with $\mathrm{PDH}$ synthesis. Furthermore, the anaerobic repression was mediated by ArcA, as witnessed by the fivefold anaerobic derepression (and up to twofold aerobic derepression) in an $\operatorname{arcA}$ mutant (Table 3). The potential for further aerobic derepression indicates that $P_{l p d}$, like other citric acid cycle promoters, is poised in a partially repressed state in aerobic cultures, such that it can be further derepressed by $\operatorname{arcA}$ mutation, and repressed under all conditions in strains containing a multicopy $\operatorname{arcA} A^{+}$ plasmid. In contrast, inactivation of the $f n r$ gene had no significant effect on expression of the $l p d A-l a c Z$ fusion, but expression was repressed by the multicopy $\mathrm{fnr}^{+}$ plasmid, suggesting that excess FNR can either invade the promoter and impair transcription or alternatively function indirectly by activating ArcA synthesis (Compan \& Touati, 1994). The role of CRP is difficult to assess; it is certainly not serving as a typical cAMPdependent activator. On the contrary, it seems to be responsible for a negative regulatory effect which is only apparent in the presence of glucose or in multicopy $\mathrm{crp}^{+}$ situations (Table 3). These effects may be indirect, and it is concluded that neither CRP nor FruR is responsible for the observed repression by glucose. Inactivation of the other regulatory genes tested (fis, rpoS and $\operatorname{bim} A$ ) revealed that Fis is a potential activator of $l p d A$ gene expression (Table 3). There is a potential Fis-binding site, GTTTAAAAATTGtTa, centred at -29 in the $l p d$ promoter region (co-ordinates 5770-5784, Stephens et al., 1983), which is mismatched at only two positions relative to the 15 bp consensus, $\mathrm{GNt}[\mathrm{c} / \mathrm{t}][\mathrm{A} / \mathrm{g}] \mathrm{a}[\mathrm{a} / \mathrm{t}]-$ $[\mathrm{T} / \mathrm{A}][\mathrm{t} / \mathrm{a}] \mathrm{tT}[\mathrm{g} / \mathrm{c}]$ anc (Finkel \& Johnson, 1992). However, Fis-binding at this position would be expected to repress, rather than activate, transcription.

\section{Transcript analysis of the SUCABCD genes by Northern blot hybridization}

The lack of correlation between sucA-lacZ expression and ODH enzyme synthesis revived an earlier suggestion that expression of the $s u c$ genes may be regulated at the upstream $s d h$ promoter (Fig. 1). This view was strengthened because the regulatory profile of the $l p d$ promoter more closely resembled that of the $s d h$ promoter than the suc promoter, and thus provided a plausible mechanism for co-expression of the $l p d A$ and $s u c A B$ genes based on shared features of the $l p d$ and $s d h$ promoters. Direct evidence was therefore sought for transcription across the $s d b B-s u c A$ region containing the suc promoter, even though no transcript had been detected by nuclease S1 mapping. Northern blot hybridization was used with a series of gene-specific probes containing parts of the $s d b C$, sucA,B,C and $D$ genes (probes $S, A, B, C$ and $D$; Fig. 1 ) and RNA extracted from two RNase mutants and the corresponding parental strains (P90C and N3433).

Hybridization with RNA from the parental strains. Using samples of parental RNA, one major band of $3.5 \mathrm{~kb}$, presumed to be the $s d h C D A B$ transcript extending from $P_{s d h}$ to the $s d h B-s u c A$ intergenic region, was detected with the $s d h$ probe ( $S$; Fig. 2). Another major band of $6.6 \mathrm{~kb}$, corresponding to a sucABCD transcript, was detected with each of the four suc gene probes $(A-D$; Fig. 2). All of the probes hybridized non-specifically to the rRNA components and several other specific bands were detected with parental RNA (Fig. 2). The $4.4 \mathrm{~kb}$ band best corresponds to a specific $s u c A B$ transcript, generated by transcript termination or processing within the $s u c B-s u c C$ intergenic region. Evidence for such termination, as well as readthrough transcription across this region, was obtained previously (Spencer \& Guest, $1985)$. A potential $3.6 \mathrm{~kb} s u c B C D$ transcript was also detected with the $s u c B, s u c C$ and $s u c D$ probes $(B-D$; Fig. 2) and there was a very weakly hybridizing $2 \cdot 1 \mathrm{~kb}$ component having $1-10 \%$ of the intensity of the $3.6 \mathrm{~kb}$ transcript, possibly representing a sucCD transcript or processed fragment (not visible in Fig. 2). However, no transcript corresponding to the entire $s d b C D A B$ sucABCD region was detected in parental RNA samples, even after prolonged exposure. This could mean that the two gene clusters are not co-transcribed or that the relevant transcript is very short-lived. It should also be stressed that it is not possible to discriminate between transcripts synthesized from an internal promoter, e.g. $P_{s u c}$, and those derived from longer transcripts by processing or degradation.

Studies with RNA from an rnc mutant. Samples of RNA from a mutant lacking RNaseIII (RS6521, rnc) were analysed with the same series of probes. This produced markedly different hybridization patterns, whose interpretation was to some extent complicated by the presence of similarly sized fragments of differing origin. Most significant was the $10 \mathrm{~kb}$ transcript detected with the $s d h$ probe after prolonged exposure of the autoradiographs (Fig. 2). It provided the first direct evidence for the existence of a full-length $s d h C D A B-s u c A B C D$ transcript. As a result, most of the other RNA molecules can be regarded as processed or degradation products of the full-length transcript. The full-length transcript was accompanied by a strong $6.5 \mathrm{~kb}$ band in addition to the $3.5 \mathrm{~kb} s d h C D A B$ transcript observed above with RNA from the parental strain. It was inferred that the $6.5 \mathrm{~kb}$ transcript extends from $P_{s d h}$ to the $3^{\prime}$ end of the sucA coding region $(s d b C-s u c A)$; this transcript would comigrate with the sucABCD transcript when parental RNA is hybridized with probe A (Fig. 2), but it is not clear why the $s d h C-s u c A$ transcript was not detected by probe $\mathrm{A}$ in the $r n c$ mutant.

The $10 \mathrm{~kb}$ full-length $s d b-s u c$ transcript was detected with all of the suc probes after prolonged auto- 

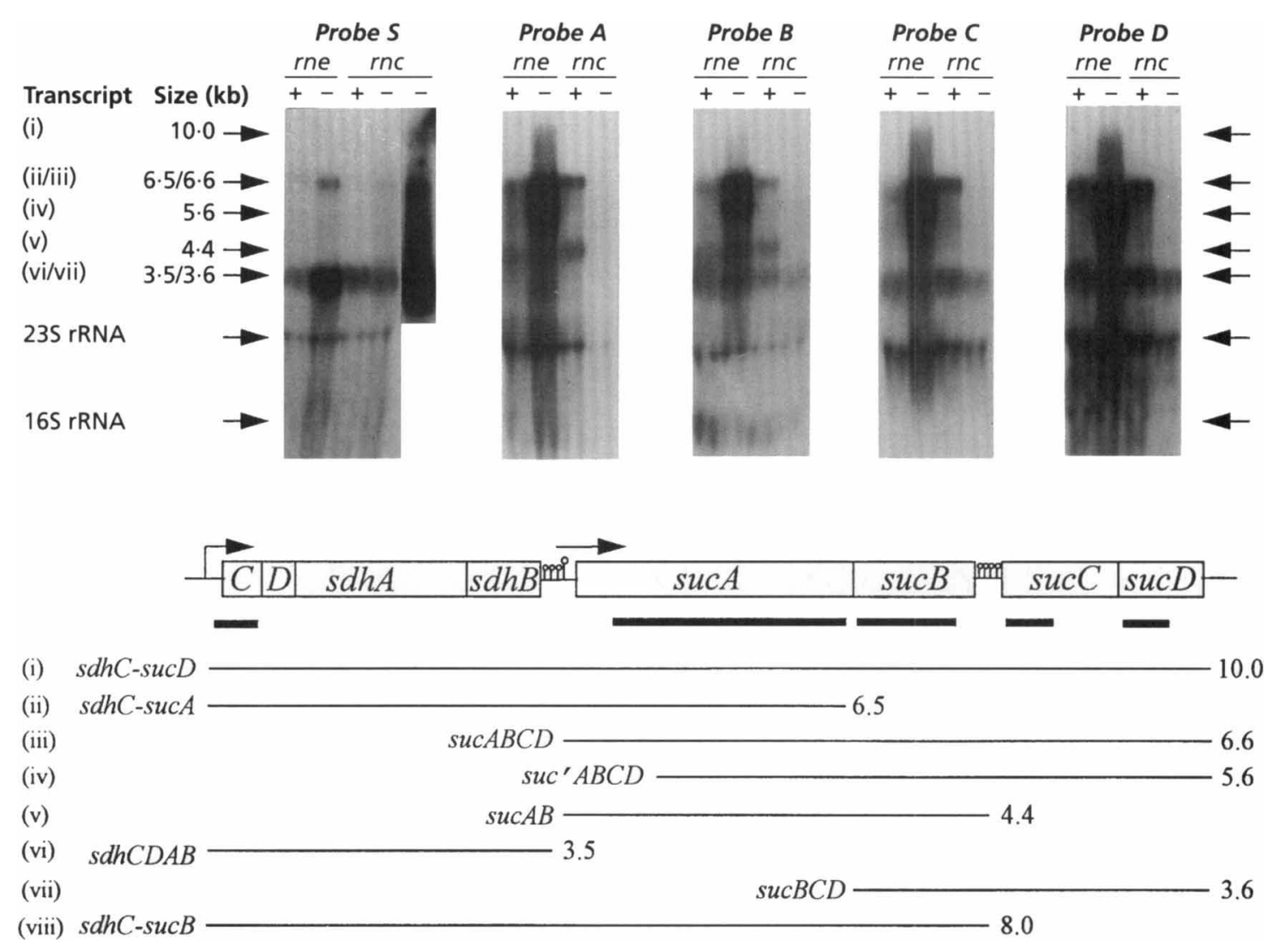

Fig. 2. Northern blot hybridization showing the transcripts detected with specific probes for citric acid cycle genes. RNA from rne and rnc mutants and the corresponding parental strains was used with probe $S$ (sdh), probe $A$ (sucA), probe B (sucB), probe $C$ (sucC) and probe $D$ (sucD). An extra track is included to show the $10 \mathrm{~kb}$ transcript detected after prolonged exposure of a hybridization of RNA from the rnc mutant with probe $S$. The origins deduced for different RNA species (denoted by lower-case Roman numerals) are shown below the map of sdh-suc operon.

radiography (data not shown). Using the same probes with RNA from the $r n c$ mutant, the $6.6 \mathrm{~kb}$ sucABCD and $4.4 \mathrm{~kb} s u c A B$ components that were prominent in parental RNA could not be detected (Fig. 2). This indicates that RNaseIII is responsible for their formation, by cleavage in the $s d h B-s u c A$ intergenic region. Retention of the $3.5 \mathrm{~kb} s d b C D A B$ transcript can be explained if a proportion of the transcripts initiating at the $s d h$ promoter are terminated at a natural termination site(s) located immediately downstream of the $s d h B$ gene, before traversing the $s d b B-s u c A$ intergenic region. The $s u c B, C$ and $D$ probes showed that the $3.6 \mathrm{~kb}$ $s u c B C D$ transcript is retained as a major component in $r n c$ mutant RNA as well as parental samples. The $3.6 \mathrm{~kb}$ $s u c B C D$ and $6.5 \mathrm{~kb} s d h C-s u c A$ transcripts are presumably derived from the full-length transcript by specific endonucleolytic cleavage at or near the sucAsucB gene boundary; the presence of both fragments in RNA from the $r n c$ mutant clearly indicates that RNaseIII is not responsible for their production (Fig. 2).

Studies with RNA from an rne mutant. There was a general increase in the relative amounts of the transcripts detected in analogous hybrization studies with RNA from the mutant lacking RNaseE (Fig. 2). This is because the overall rate of mRNA decay is lowered when
RNaseE is inactivated. All of the transcripts observed with RNA from the parental strains were present in RNA from the rne mutant. In addition, the $10 \mathrm{~kb}$ fulllength transcript could be detected with all of the probes (including the $s d b$ probe after longer exposure than shown in Fig. 2), presumably due to general transcript stabilization. Some new components, which probably represent decay intermediates, were also observed, e.g. the $5.6 \mathrm{~kb} s u c^{\prime} A B C D$ fragment detected by all of the suc probes and a potential $3.4 \mathrm{~kb} s u c^{\prime} A B$ transcript detected by the $s u c A$ and $s u c B$ probes (Fig. 2).

Transcript stabilities. The stabilities of the four major transcription products were investigated by quantifying the temporal changes that occurred after adding rifampicin to block transcript initiation (Fig. 3). The relative amount of the $s d b C D A B$ transcript declined at a rate corresponding to a chemical half-life of $3.3 \mathrm{~min}$. However, in the case of the sucABCD transcript and its subfragments, the relative amounts increased for up to $4 \mathrm{~min}$ before declining with half-lives of $3.6 \mathrm{~min}$ for the sucABCD and $s u c A B$ transcripts, and $5.4 \mathrm{~min}$ for the $s u c B C D$ transcript. The reason for this behaviour is not clear. It would appear that a significant proportion of the suc transcripts is for some reason not accessible to hybridization, or not formed until after the rifampicin is 


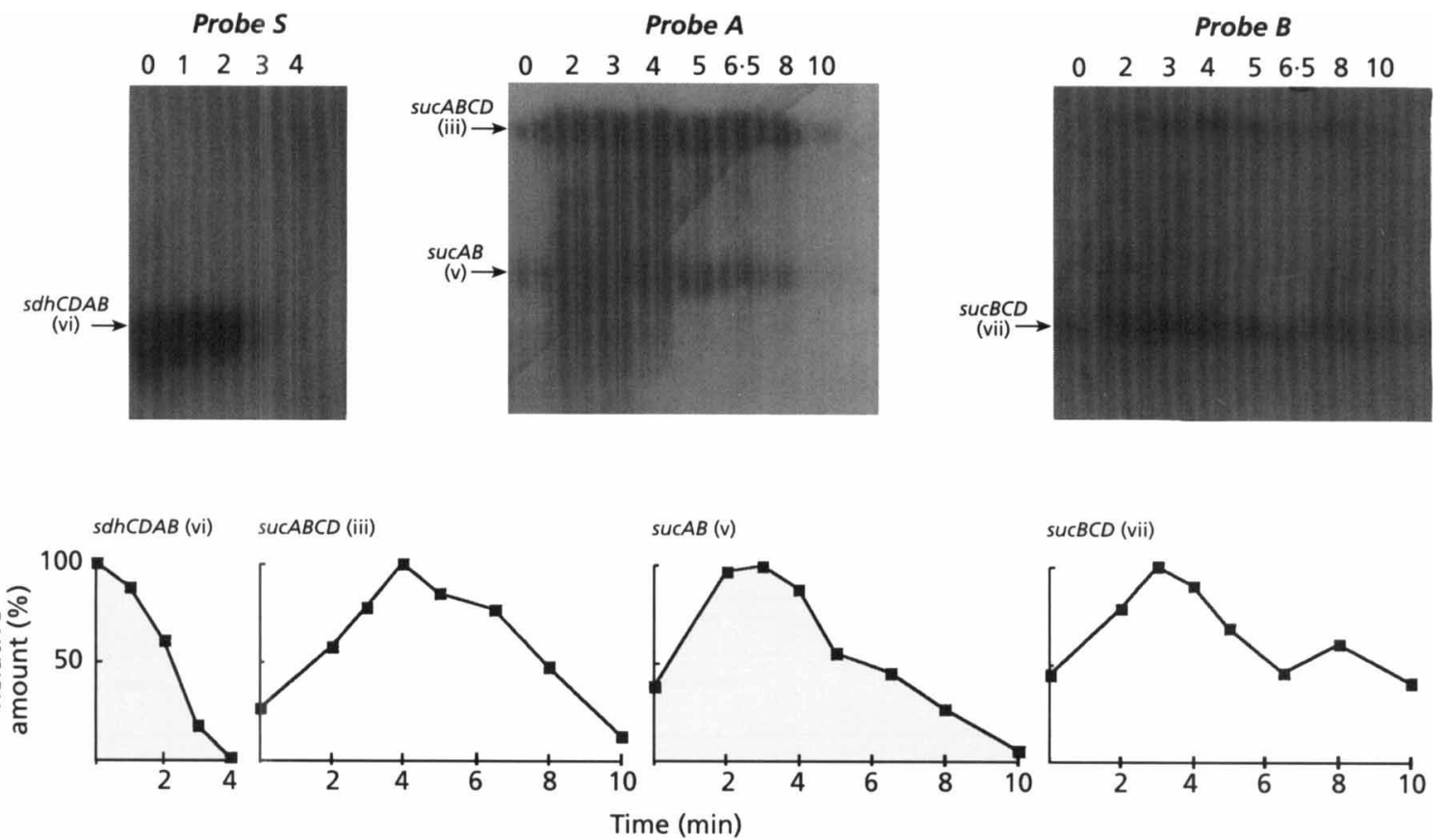

Fig. 3. Northern blots showing temporal changes in different RNA species after blocking transcript initiation. Three genespecific probes (S, sdh; $A$, sucA; and $B$, sucB) were used with RNA extracted from W3110 at different times after adding rifampicin $(200 \mu \mathrm{g} \mathrm{ml}-1)$. The amounts of the four major RNA species, derived by densitometric analysis of autoradiographs, were expressed as percentages of the maximum amount of each species and plotted versus time.

added. The turnover rates may be such that suc transcripts are generated after rifampicin addition by active RNA polymerase molecules that are stalled for example, in the $s d h B-s u c A$ intergenic region.

\section{Primer extension analysis}

Primer extension analysis was used to define the $5^{\prime}$ extremities of the major transcription products encoding suc genes. Three distinct start-sites or processing sites (A, B and C) were detected in the $s d b B-s u c A$ intergenic region using $S 410$ as primer (Fig. 1) with RNA from the two parental strains (Fig. 4a). The same products were detected with increased intensity in RNA from the rne mutant but, very significantly, none were detected with RNA from the $r n c$ mutant (Fig. 4a). This indicates that all three ends are generated by RNaseIII cleavage in the $s d b-s u c$ intergenic region. The existence of three RNaseIII cleavage sites explains why transcripts spanning the $s d h-s u c$ intergenic region were so difficult to detect. Their precise locations are shown in Fig. 4(b) and it is interesting to note that sites $A$ and $B$ are within $3 \mathrm{bp}$ and $1 \mathrm{bp}$ of the putative sucA transcriptional start-sites identified previously by the 'less precise nuclease $S 1$ mapping procedure (Spencer \& Guest, 1985). It is not known whether one of the less intense products generated with primer $\mathrm{S} 410$ corresponds to the start-site of the independent sucA promoter. Such a site should have been more apparent with RNA from the $r n c$ mutant, especially if it is located upstream of one or more of the RNaseIII processing sites. Unfortunately, potential sucA start-sites are masked by the minor artefactual bands which are generated by primer extension in regions of RNA secondary structure, and there are no recognizable promoter sequences in the $s d h-s u c$ intergenic region, which is consistent with the weak activity of the sucA promoter.

Analogous studies with RNA from both mutant and parental strains and primers spanning the sucA coding and $s u c A-s u c B$ intergenic regions (S459, S462-469; Fig. 1) revealed two $5^{\prime}$ extremities within the sucA coding region, but no start-sites or processing sites were detected immediately upstream of the $s u c B$ gene (data not shown). The internal $s u c A$ sites were $1.3 \mathrm{~kb}$ and $1.8 \mathrm{~kb}$ from the beginning of the sucA gene, and both were detected in parental RNA and more prominent in rne mutant RNA. Specific cleavage at the first site would generate the $5.6 \mathrm{~kb} s u c^{\prime} A B C D$ fragment (Fig. 2). No specific cleavage site was detected in or near the $s u c A-s u c B$ junction, needed to explain the origin of the $6.5 \mathrm{~kb} s d h C-s u c A$ and $3.6 \mathrm{~kb} s u c B C D$ fragments.

Primer extension analysis with primer S407 (Fig. 1) revealed a series of putative $5^{\prime}$ ends in the $s u c B-s u c C$ intergenic region and in the distal segment of the $s u c B$ coding region (data not shown). These were probably due to pausing of the reverse transcriptase in the region containing four REP sequences rather than to specific initiation or processing events. The products were more intense in the $r n c$ and rne mutants than in the parents, but the significance of this is not known. Transcription was previously shown to read across the sucB-sucC 
(a)

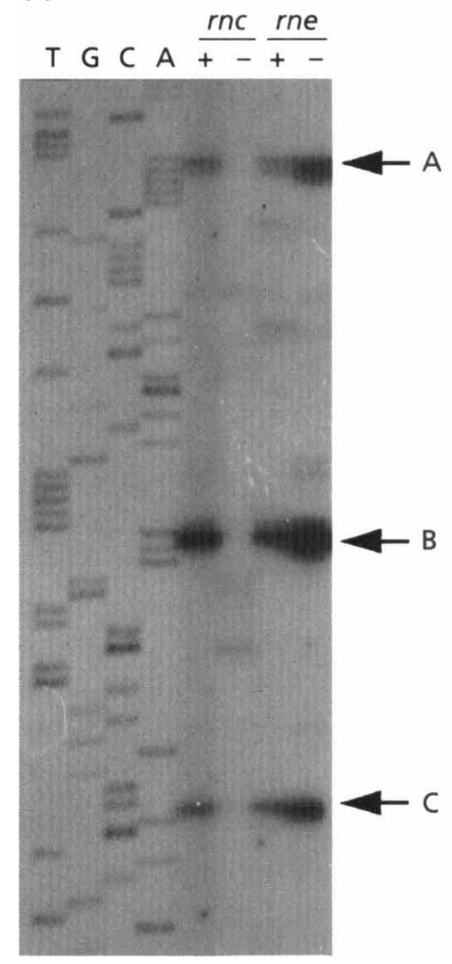

(b)

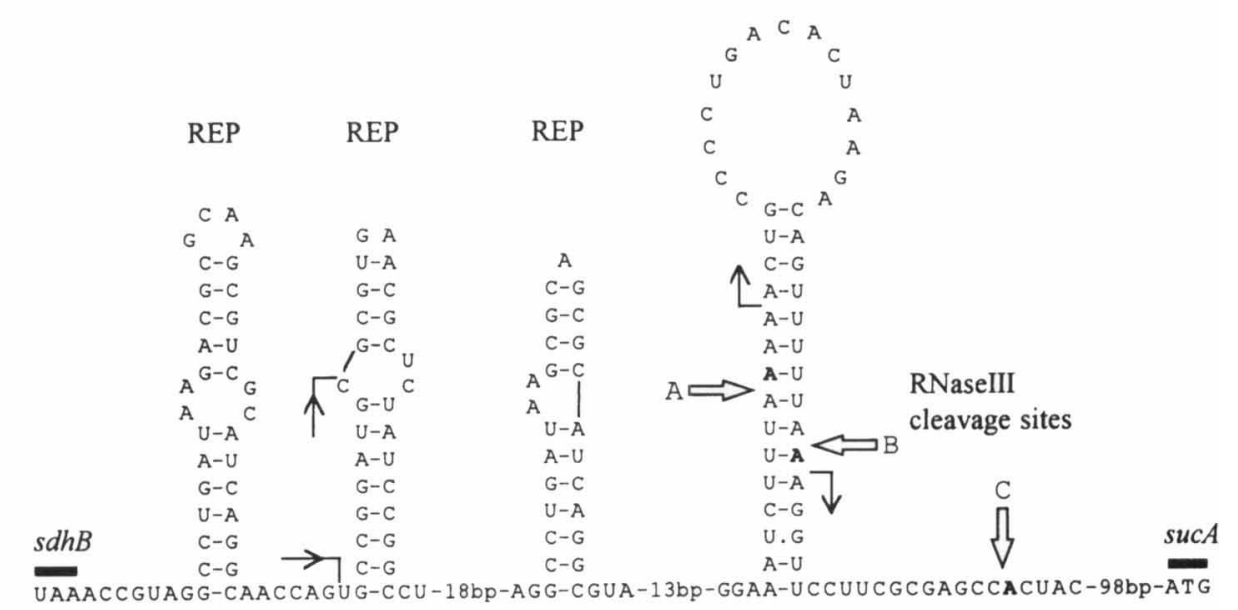

Fig. 4. Primer extension mapping and location of RNaselll processing sites in the sdhB-sucA intergenic region. (a) Primer extension analyses with RNA extracted from rne and rnc mutants and the corresponding parental strains, aligned to a sequence ladder generated with the same primer. The major products are denoted by the arrows, $A, B$ and $C$. There is a $0.5 \mathrm{bp}$ discrepancy between the primer extension products and the sequence ladder, but the validity of the interpretation is supported by other experiments. (b) Details of the sdhB-sucA intergenic region showing the positions of three RNaselll processing sites $(A-C)$ deduced from the primer extension products detected in (a). The positions of three REP sequences, another potential stem-loop, and the sdh transcript end points $(\rightarrow)$ and sucA transcript start-sites $(\rightarrow)$ defined previously by $\$ 1$ mapping (Spencer \& Guest, 1985), are shown.

intergenic region, and although a termination or processing site was identified 220 bp downstream of $s u c B$, no evidence for the existence of an independent sucC promoter was found (Spencer \& Guest, 1985). There is no potential promoter sequence in the intergenic region and the corresponding sucC-lac $Z$ fusions are inactive (Park et al., 1997; data not shown).

\section{DISCUSSION}

The lacZ fusion studies showed that the regulatory profile of the suc promoter bears virtually no resemblance to those of the corresponding enzymes $(\mathrm{ODH}$ and SCS) or the $l p d$ promoter. Compared to the lpd promoter, there was no ArcA- or FNR-dependent anaerobic repression, no glucose repression, nor any other common feature that could provide a mechanism for co-regulating sucABCD and $l p d A$ expression. This contrasts with the features shared by the regulatory profiles of the $s d h$ promoter (Iuchi \& Lin, 1988; Iuchi et al., 1994; Park et al., 1995) and the $l p d$ promoter, and synthesis of the corresponding enzymes ( $\mathrm{SDH}, \mathrm{ODH}$ and SCS). Indeed, the similarities provide strong support for the view that sucABCD transcription is initiated and regulated primarily from the $s d h$ promoter, and this in turn provides a plausible mechanism co-ordinating expression of the $s u c A B$ and $l p d A$ genes for synthesis of the ODH complex. Direct support for co-transcription of the $s d h$ and $s u c$ genes has now been obtained by detecting a full-length $s d h C D A B-s u c A B C D$ transcript and independently from parallel studies with a sdbCDAB-sucA-lacZ fusion (Park et al., 1997). This raises questions concerning which factors are primarily responsible for co-regulating the $l p d$ and $s d h$ promoters, and what is the normal role of the suc promoter.

In addition to being repressed by anaerobiosis and glucose the $s d h$ promoter is more active in the absence of Fis and $\sigma^{38}(\mathrm{Xu} \&$ Johnson, 1995) whereas the lpd promoter is activated by Fis and unaffected by $\sigma^{38}$. The regulatory coupling of the $s d h$ and $l p d$ promoters would thus appear to depend primarily on their ArcA-mediated anaerobic repression. The co-regulatory importance of glucose-mediated repression is uncertain because different mechanisms may be involved at each of the two promoters. Early work with an $s d h-g a l K$ fusion had indicated that crp and cya are required for maximum expression, and S1 mapping studies not only confirmed that $s d h$ transcription is severely repressed by glucose but indicated that there is a potential CRP-binding site 
at -80 in the $s d b$ promoter region (Wilde \& Guest, 1986). This promoter and its CRP-binding site can now be deemed responsible for the co-ordinate expression and the glucose-mediated repression of ODH and SCS synthesis observed by Buck et al. (1986). Indeed, the absence of a CRP-binding site in the suc promoter region was used to support the early suggestion that the $s d h$ and $s u c$ genes are co-transcribed. In contrast, the $l p d$ promoter is not CRP-dependent nor is it associated with a potential CRP-binding site, yet it is partially repressed by glucose (Table 3 ). Recent work has likewise shown that the effect of glucose on $s d h$ transcription is not mediated by CRP or FruR (Park et al., 1995). This means that some alternative mechanism may be co-regulating the $s d h$, lpd (and the independent suc) promoters. However, the need for coupling in response to glucose availability is uncertain, because transcription of the $l p d A$ gene from the CRP- and glucose-independent $p d b$ promoter probably supplies excess E3 subunits, due to the E3 content of the PDH complex being approximately half that of the other two components.

The regulatory features of the suc promoter, lack of anaerobic repression and activation by $\sigma^{38}$, suggest that it may provide E1o, E2o and SCS during anaerobic growth and in stationary phase, when the $s d h$ promoter is severely repressed and some SDH functions are replaced by fumarate reductase. Indeed, a slight anaerobic activation of the sucA promoter was observed by Park et al. (1997). The corresponding enzyme activities are presumably needed under anaerobic conditions to provide succinic semialdehyde-TPP (from 2oxoglutarate) for menaquinone biosynthesis (Meganathan, 1996) and to provide succinyl-CoA (from either succinate or 2-oxoglutarate) for use in diaminopimelate and lysine biosynthesis (Patte, 1996) and methionine biosynthesis (Greene, 1996). The present location of the $s u c A B$ genes could mean they were inserted into an ancestral sdhCDAB-sucCD (SDH-SCS) operon during the evolution of the citric acid cycle or that this organization facilitates switching between the cyclic and non-cyclic forms of the citric acid cycle associated with the aerobic and anaerobic metabolic modes adopted by $E$. coli. If so, the suc promoter may be a vestigial remnant of an ancestral suc operon, that may retain specific anaerobic roles to allow some differential expression relative to the $s d h$ promoter.

Detection of the full-length $s d b C D A B-s u c A B C D$ transcript, albeit only in $r n c$ and rne mutants, has now established that the eight genes encoding three citric acid cycle enzymes are co-transcribed from the $s d h$ promoter. The operon contains one internal promoter $\left(P_{s u c}\right)$ and termination can occur downstream of the $s d b B$ and $s u c B$ genes in the intergenic regions that contain REP elements (Fig. 1). The extreme lability of the readthrough transcript is attributed to RNaseIII-mediated cleavage at one or more of three sites in the $s d h B-s u c A$ intergenic region. This intergenic region is potentially rich in double-stranded RNA and hence targets for RNaseIII (Fig. 4b). Indeed, the asymmetric organization of the cleavage sites A and B conforms to that of the RNaseIII cleavage-site consensus (Krinke \& Wulff, 1990). This does not apply to site $C$, which is seemingly located in a single-stranded region. It is important to note that even in the $r n c$ mutant, the $3.5 \mathrm{~kb} s d h C D A B$ transcript is generated, presumably by natural termination at the previously identified sites (Spencer \& Guest, 1985) located upstream of the RNaseIII processing sites (Fig. $4 \mathrm{~b})$. The $s u c B-s u c C$ intergenic region is similarly rich in potential secondary structure but the terminator or processing site is located downstream of the REP sequences (Spencer \& Guest, 1985) and RNaseIII is not involved here. The role of $\mathrm{RNaseE}$ in processing transcripts of the $s d b D C A B-s u c A B C D$ operon is less defined. The general increase of stability of the major transcripts, and the presence of extra components in RNA from the rne mutant, show that RNaseE is involved in transcript processing and degradation, but the sequence specificity of this enzyme is still unknown (Cohen \& McDowall, 1997). The degradation of mRNA by RNaseIII and RNaseE is an important factor in defining the functional life of a transcription product (Kushner, 1996). In the case of the sdbCDAB-sucABCD operon it will in future be important to define the relative contributions of transcription initiation, transcription termination and transcript degradation to the overall patterns of enzyme synthesis under different conditions. It would be particularly interesting to understand the mechanism controlling natural termination relative to readthrough transcription at the internal sites and its physiological significance.

\section{ACKNOWLEDGEMENTS}

We are very grateful to $\mathrm{Dr}$ J. Green for helpful discussions. This work was supported by The Wellcome Trust.

\section{REFERENCES}

Aiba, H., Adhya, S. \& de Crombrugghe, B. (1981). Evidence for two functional gal promoters in intact Escherichia coli cells. J Biol Chem 256, 11905-11910.

Amarasingham, C. R. \& Davis, B. D. (1965). Regulation of $\alpha$ ketoglutarate dehydrogenase formation in Escherichia coli. J Biol Chem 240, 3664-3668.

Ball, C. A., Osuna, R., Ferguson, K. C. \& Johnson, R. C. (1992). Dramatic changes in fis levels upon nutrient upshift in Escherichia coli. J Bacteriol 174, 8043-8056.

Bonnefoy, V., Pascal, M.-C., Ratouchniak, J. \& Chippaux, M. (1986). Alteration by mutation of the control by oxygen of the nar operon in Escherichia coli. Mol Gen Genet 205, 349-352.

Bradford, M. M. (1976). A rapid and sensitive method for the quantitation of microgram quantities of protein utilising the principle of protein-dye binding. Anal Biochem 72, 248-253.

Buck, D., Spencer, M. E. \& Guest, J. R. (1986). Cloning and expression of the succinyl-CoA synthetase genes of Escherichia coli K12. J Gen Microbiol 132, 1753-1762.

Buck, M., Woodcock, J., Cannon, W., Mitchenall, L. \& Drummond, M. (1987). Positional requirements for the function of NIFspecific upstream activator sequences. Mol Gen Genet 210, 140-144.

Cohen, S. N. \& McDowall, K. J. (1997). RNaseE: still a wonderfully mysterious enzyme. Mol Microbiol 23, 1099-1106. 
Compan, I. \& Touati, D. (1994). Anaerobic activation of $\operatorname{arcA}$ transcription in Escherichia coli: roles for Fnr and ArcA. Mol Microbiol 11, 955-964.

Cronan, J. E., Jr \& LaPorte, D. (1996). Tricarboxylic acid cycle and glyoxylate bypass. In Escherichia coli and Salmonella typhimurium: Cellular and Molecular Biology, pp. 206-216. Edited by F. C. Neidhardt and others. Washington, DC: American Society for Microbiology.

Cunningham, L., Gruer, M. J. \& Guest, J. R. (1997). Transcriptional regulation of the aconitase genes $(a c n A$ and $a c n B)$ of Escherichia coli. Microbiology 143, 3795-3805.

Finkel, S. E. \& Johnson, R. C. (1992). The Fis protein: it's not just for DNA inversion anymore. Mol Microbiol 6, 3257-3265.

Gerischer, U. \& Durre, P. (1992). mRNA analysis of the adc gene region of Clostridium acetobutylicum during the shift to solventogenesis. J Bacteriol 174, 426-433.

Goldblum, K. \& Apirion, D. (1981). Inactivation of the ribonucleic acid-processing enzyme ribonuclease $\mathrm{E}$ blocks cell division. $J$ Bacteriol 146, 128-132.

Greene, R. C. (1996). Biosynthesis of methionine. In Escherichia coli and Salmonella typhimurium: Cellular and Molecular Biology, pp. 542-560. Edited by F. C. Neidhardt and others. Washington, DC: American Society for Microbiology.

Guest, J. R. \& Russell, G. C. (1992). Complexes and complexities of the citric acid cycle in Escherichia coli. Curr Top Cell Regulation 33, 231-247.

Guest, J. R., Angier, S. J. \& Russell, G. C. (1989). Structure, expression and protein engineering of the pyruvate dehydrogenase complex of Escherichia coli. Ann N Y Acad Sci 573, 76-99.

luchi, S. \& Lin, E. C. C. (1988). arcA (dye), a global regulatory gene in Escherichia coli mediating repression of enzymes in aerobic pathways. Proc Natl Acad Sci USA 85, 1888-1892.

luchi, S., Aristarkov, A., Dong, J. M., Taylor, J. S. \& Lin, E. C. C. (1994). Effects of nitrate respiration on expression of the Arccontrolled operons encoding succinate dehydrogenase and flavinlinked L-lactate dehydrogenase. J Bacteriol 176, 1695-1701.

Krinke, L. \& Wulff, D. L. (1990). The cleavage specificity of RNaseIII. Nucleic Acids Res 18, 4809-4815.

Kushner, S. R. (1996). mRNA decay. In Escherichia coli and Salmonella typhimurium: Cellular and Molecular Biology, pp. 849-860. Edited by F. C. Neidhardt and others. Washington, DC: American Society for Microbiology.

Meganathan, R. (1996). Biosynthesis of the isoprenoid quinones menaquinone (vitamin $\mathrm{K}_{2}$ ) and ubiquinone (Coenzyme Q). In Escherichia coli and Salmonella typhimurium: Cellular and Molecular Biology, pp. 642-664. Edited by F. C. Neidhardt and others. Washington, DC: American Society for Microbiology.

Miller, J. H. (1972). Experiments in Molecular Genetics. Cold Spring Harbor, NY: Cold Spring Harbor Laboratory.

Miller, J. H. \& Albertini, A. M. (1983). Effects of surrounding sequence on the suppression of nonsense codons. J Mol Biol 164, 59-71.

Park, S.-J., Tseng, C.-P. \& Gunsalus, R. P. (1995). Regulation of succinate dehydrogenase $(s d h C D A B)$ operon expression in Escherichia coli in response to carbon supply and anaerobiosis: role of ArcA and Fnr. Mol Microbiol 15, 473-482.

Park, S.-J., Chao, G. \& Gunsalus, R. P. (1997). Aerobic regulation of the sucABCD genes of Escherichia coli, which encode $\alpha$ ketoglutarate dehydrogenase and succinyl coenzyme A syn- thetase: roles of $\operatorname{ArcA}, \mathrm{Fnr}$, and the upstream $s d h C D A B$ promoter. J Bacteriol 179, 4138-4142.

Patte, J.-C. (1996). Biosynthesis of threonine and lysine. In Escherichia coli and Salmonella typhimurium: Cellular and Molecular Biology, pp. 528-541. Edited by F. C. Neidhardt and others. Washington, DC: American Society for Microbiology.

Perham, R. N. (1991). Domains, motifs and linkers in 2-oxo acid dehydrogenase multienzyme complexes : a paradigm in the design of a multifunctional protein. Biochemistry 30, 8501-8512.

Philips-Jones, M. K., Watson, F. J. \& Martin, R. (1993). The 3' codon effect on UAG suppressor tRNA in different Escherichia coli and human cells. $J$ Mol Biol 233, 1-6.

Quail, M. A., Haydon, D. J. \& Guest, J. R. (1994). The $p d h R-$ aceEF-lpd operon of Escherichia coli expresses the pyruvate dehydrogenase complex. Mol Microbiol 12, 95-104.

Sambrook, J., Fritsch, E. F. \& Maniatis, T. (1989). Molecular Cloning: a Laboratory Manual, 2nd edn. Cold Spring Harbor, NY: Cold Spring Harbor Laboratory.

Schroder, I., Darie, S. \& Gunsalus, R. P. (1993). Activation of the Escherichia coli nitrate reductase (narGHJI) operon by NarL and Fnr requires integration host factor. $J$ Biol Chem 268, 771-774.

Silhavy, T. J., Barman, M. L. \& Enquist, L. W. (1984). Experiments with Gene Fusions. Cold Spring Harbor, NY: Cold Spring Harbor Laboratory.

Simons, R. W., Houman, F. \& Kleckner, N. (1987). Improved single and multicopy lac-based cloning vectors for protein and operon fusions. Gene 53, 85-96.

Smith, M. W. \& Neidhardt, F. C. (1983). 2-Oxoacid dehydrogenase complexes of Escherichia coli: cellullar amounts and patterns of synthesis. J Bacteriol 156, 81-88.

Spencer, M. E. \& Guest, J. R. (1982). Molecular cloning of four tricarboxylic acid cycle genes of Escherichia coli. J Bacteriol 151, 542-552.

Spencer, M. E. \& Guest, J. R. (1985). Transcription analysis of the $s u c A B, a c e E F$ and $l p d$ genes of Escherichia coli. Mol Gen Genet 200, 145-154.

Spencer, M. E. \& Guest, J. R. (1987). Regulation of citric acid cycle genes in facultative bacteria. Microbiol Sci 4, 164-168.

Spencer, M. E., Wilde, R. J. \& Guest, J. R. (1986). Transcription and regulation of TCA cycle genes of E. coli. Soc Gen Microbiol Q 13, M9.

Spiro, S., Roberts, R. E. \& Guest, J. R. (1989). FNR-dependent repression of the $n d h$ gene of Escherichia coli and metal ion requirement for FNR-regulated gene expression. Mol Microbiol 3, 601-608.

Stephens, P. E., Lewis, H. M., Darlison, M. G. \& Guest, J. R. (1983). Nucleotide sequence of the lipoamide dehydrogenase gene of Escherichia coli K12. Eur J Chem 135, 519-527.

Wilde, R. J. \& Guest, J. R. (1986). Transcript analysis of the citrate synthase and succinate dehydrogenase genes of Escherichia coli K12. J Gen Microbiol 132, 3239-3251.

Xu, J. \& Johnson, R. C. (1995). Identification of genes negatively regulated by Fis: Fis and RpoS comodulate growth-phasedependent gene expression in Escherichia coli. J Bacteriol 177, 938-947.

Received 2 February 1998; revised 24 March 1998; accepted 17 April 1998. 